\title{
Análisis de sensibilidad de sistemas fotovoltaicos conectados a la red eléctrica: Caso de estudio en Guerrero, México
}

\section{Sensitivity analysis of grid-connected photovoltaic systems: Case study in Guerrero, Mexico}

Rivera-Martínez Mario Arturo

Universidad Politécnica del Estado de Guerrero, Taxco de Alarcón

Correo: marioarturo64@gmail.com

Hernández-Galvez Geovanni (Autor correspondiente)

Universidad Popular de la Chontalpa, Tabasco

Correo: geovannisg@yahoo.com

Sarracino-Martínez Omar

Universidad Popular de la Chontalpa, Tabasco

Correo: omar_sarra@hotmail.com

Ixtlilco-Cortés Luis

Universidad Politécnica del Estado de Guerrero, Taxco de Alarcón

Correo: ixtlilco@hotmail.com
Juantorena-Ugás Alina

Universidad Autónoma del Estado de Morelos

Correo: alinajt2@yahoo.es

Batalla-Nolasco Ulises Ivan

Universidad Politécnica del Estado de Guerrero, Taxco de Alarcón Correo: black_eyed_banu@hotmail.com

Becerra-García David

Universidad Politécnica del Estado de Guerrero, Taxco de Alarcón Correo: davidbega@yahoo.com

\section{Resumen}

Se realiza un análisis de Sensibilidad de Sistemas Fotovoltaicos (SFV) enlazados a la red eléctrica a partir de un caso de estudio en la UPEG. Se determinó la influencia de las siguientes variables en el sistema FV óptimo: costos capitales y de reemplazamiento de los módulos FV; precio de la electricidad comprada a CFE; tasa de interés anual; carga promedio de consumo y radiación solar promedio diaria. Las tres primeras se incluyeron porque la Reforma Energética en México les podría causar modificaciones importantes. Para los precios actuales de los componentes fotovoltaicos en México y $6 \%$ de tasa de interés, un sistema con una potencia de $50 \mathrm{~kW}$ podría abastecer 50\% de la demanda de la UPEG, a un costo nivelado de 0.27 USD/kWh; mientras que uno de $100 \mathrm{~kW}$ abastecería 80\% de la demanda a un costo de 0.20 USD/kWh. Sin embargo, si el precio de la electricidad comprada a CFE disminuyera por debajo de los 0.25 USD/kWh, la alternativa fotovoltaica dejaría de ser competitiva. En dicho escenario los SFV solo serían competitivos para tasas de interés alrededor de 3\%; pero si el costo capital FV disminuyera en un 50\% serían competitivos incluso a tasas de interés de $6 \%$ y costos de la electricidad proveniente de CFE no menores a 0.15 USD $\$ / \mathrm{kWh}$. Adicionalmente, los periodos de recuperación mostraron valores relativamente altos.

Descriptores: Tecnologías renovables, sistemas fotovoltaicos, optimización de sistemas fotovoltaicos, autoabastecimiento energético, sensibilidad de sistemas interconectados.

\begin{abstract}
A sensitivity analysis of grid-connected Photovoltaic Systems (PVS) was realized in a specific case of study at UPEG (Polytechnic University of Guerrero State). It was determined the influence of the following variables on the very best PVS: capital costs and replacement of solar panels, electrical power price consumed to CFE; annual interest rate, average charge consumption and daily average solar radiation. The first three variables were includes because the energy reform in México could cause on them crucial modifications. For the current prices of photovoltaic components in México and a $6 \%$ of interest rate, a system with $50 \mathrm{~kW}$ power could provide around 50\% of electrical demand of UPEG: a levelized cost of $0.27 \mathrm{USD} / \mathrm{kWh}$. While $100 \mathrm{~kW}$ would supply $80 \%$ of demand with a cost of 0.20 USD/kWh. Nevertheless, if the price of electricity bought to CFE was diminished below 0.25 USD/ kWh, the photovoltaic alternative would not be competitive. In that setting the PVS would only be competitive for interest rate around $3 \%$. But if the PV capital cost decreses to $50 \%$, they would be competitive even at $6 \%$ rate interest and the electricity costs from CFE not less that 0.15 USD $\$ / \mathrm{kWh}$. Adidtionally, the periods of recovery showed relatively high values.
\end{abstract}

Keywords: Renewable technologies, photovoltaic systems, optimization of photovoltaic systems, energy self-supply, sensitivity of interconnected systems. 


\section{INTRODUCCIÓN}

El cambio climático es uno de los grandes problemas mundiales sobre el que se habla cada día y del que han surgido diversas teorías, incluyendo aquellas que no lo atribuyen a causas antropogénicas, a pesar de que existen argumentos suficientes para asegurar que la actividad del hombre contribuye decididamente a ello. Este es un problema de dimensiones considerables, toda vez que la población mundial continúa creciendo y con ello la demanda de energía; principal causante de la emisión de Gases de Efecto Invernadero (GEI), que favorecen el calentamiento global. El consumo de energía primaria en el 2014 fue de 12928.8 millones de toneladas equivalentes de petróleo (Mtep), lo cual representó un aumento de $0.9 \%$ en relación con 2013 (International Energy Agency, 2014).

Adicionalmente, el petróleo aún es el combustible más utilizado a nivel mundial, lo que ha ocasionado que las emisiones de GEI aumenten considerablemente.

Ante tal situación, los gobiernos y organismos internacionales emprendieron diferentes iniciativas de mitigación de emisiones y muchos países se han trazado metas significativas de reducción. En particular, México estableció el compromiso ante la Convención Marco de las Naciones Unidas para reducir $25 \%$ sus emisiones de GEI para el 2030. Esta meta aspiracional implica retos que no son fáciles de alcanzar, pero sin duda constituye una excelente oportunidad para que desde todos los ámbitos de actuación se desarrollen iniciativas de carácter innovador, incluyentes, replicables y sobre todo sustentables que contribuyan a la protección y preservación de este planeta. Uno de esos ámbitos, es el energético, en el que ya se desarrollan importantes programas y reformas en el país; que abarcan no solo el uso de tecnologías renovables sino también la implementación de programas y medidas de eficiencia energética.

En particular, el uso de tecnologías fotovoltaicas en México creció (tabla 1) y se espera continúe así en los próximos años; este crecimiento estará influenciado por la forma en que la reforma energética, recientemente aprobada en el país, incida en el sector energético nacional. A ello contribuirán factores tecnológicos, económicos, sociales, ambientales, financieros y de políticas públicas, entre otros, que estan estrechamente relacionados, debido a los retos que impone el desarrollo energético sustentable.

En este sentido, el estudio de sistemas fotovoltaicos se aborda ampliamente por diferentes autores a nivel mundial. Sin embargo, en Mé- xico no abundan los trabajos que realicen un análisis de la influencia que podrían tener factores de diferente naturaleza en el desempeño de tales sistemas; donde la simulación y el análisis de sensibilidad son herramientas que pueden contribuir a ello, ya que a nivel mundial se utilizan bastante.

Durante la última década se han realizado numerosos estudios que modelan diferentes sistemas energéticos renovables, donde el software de modelación HOMER (Hybrid Optimization Model for Electric Renewables) es uno de los más empleados para ello. Entre dichos estudios se encuentra el realizado por Getachew y Björn (2010), quienes estudian mediante HOMER la factibilidad de un sistema híbrido eólico-fotovoltaico para una localidad remota en Etiopía y evalúan la influencia de la velocidad del viento, del costo fotovoltaico y del precio del diésel en las soluciones óptimas. Por su parte, Saheb et al. (2011) realizaron un análisis comparativo entre un sistema híbrido eólico-fotovoltaico enlazado a red y uno autónomo, para una región árida de Argelia, basado en sus impactos económicos y ambientales. Los resultados demostraron, mediante un análisis de sensibilidad, que la variable que más afectó al sistema óptimo fue la velocidad media del viento.

El modelo HOMER también se utilizó por Velo et al. (2014) para realizar el análisis económico de un sistema híbrido eólico-diésel autónomo y compararlo con la opción de utilizar solo la red eléctrica convencional. El estudio se realizó para una granja de ganado en cuatro localidades españolas con diferentes velocidades medias del viento. Por otro lado, Bronski et al. $(2014,2015)$ se apoyaron en dicho modelo para evaluar las condiciones bajo las cuales los sistemas fotovoltaicos distribuidos con sistema de almacenamiento competirían con la red eléctrica convencional en algunas regiones de Estados Unidos o qué tanto se podría sustituir con ellos el suministro de electricidad proveniente de la red.

Un estudio con propósitos similares se condujo por Khalilpour y Vassallo (2015), quienes evaluaron para ciertas condiciones de precios y niveles de consumo eléctrico si el abandono de la red (independizarse de la red eléctrica) es una "ambición" o una "opción real". En

Tabla 1. Capacidad fotovoltaica instalada en México entre 2008 y 2014 (acumulada) (International Energy Agency, 2015)

\begin{tabular}{cccccccc}
\hline Año & 2008 & 2009 & 2010 & 2011 & 2012 & 2013 & 2014 \\
\hline $\begin{array}{c}\text { Capacidad } \\
\text { acumulada } \\
\text { (MW) }\end{array}$ & 21.7 & 25.0 & 30.6 & 40.1 & 52.1 & 112.1 & 179.1 \\
\hline
\end{tabular}


dicho estudio los autores concluyeron que, para los niveles actuales de consumo, el abandono de red no es aún viable, a pesar de la continua disminución en los precios de los sistemas fotovoltaicos y las baterías. Adicionalmente concluyen que, en términos de políticas públicas, se debe evolucionar hacia un mayor incentivo de los sistemas interconectados a red, donde los consumidores también puedan jugar el papel de suministradores de energía a la misma.

Este tema también se aborda por Mudasser et al. (2015) quienes analizan la viabilidad de un sistema híbrido eólico-biogás para tres localidades en Canadá y dos esquemas de producción de energía: uno en el que la empresa eléctrica no le paga los excesos de electricidad al productor y otro en el que sí compra dichos excesos a precios diferenciados. El estudio demuestra que en el primer caso el sistema híbrido eólico-biogás, no fue económicamente factible en ninguno de los tres sitios considerados. Mientras que, al incluirse los incentivos dados por la compra de los excesos de electricidad, los sistemas mejoraron sus indicadores económicos; alcanzándo periodos de recuperación de la inversión de 4 años en uno de los sitios estudiados.

En otros estudios, Ramli et al. (2015) utilizaron el modelo HOMER para determinar el tamaño óptimo de los componentes de un sistema fotovoltaico enlazado a la red, en una localidad de Arabia Saudita. El método establecido permitió encontrar la relación óptima entre las capacidades del sistema fotovoltaico y del inversor, bajo restricciones de "cero carga no servida" y "cero exceso de electricidad". Similarmente, Kalincy (2015) simula diferentes escenarios para seleccionar la alternativa óptima de suministro de electricidad a la isla turca de Bozcaada. Los sistemas analizados incluyen tecnologías eólicas, fotovoltaicas y de hidrógeno; asimismo se modelaron utilizando la herramienta de optimización HOMER.

También se estudió un sistema híbrido eólico/fotovoltaico/biogás para electrificar viviendas rurales en el suroeste de Noruega. En dicho estudio, realizado por Sarker (2016), se realizó un análisis de sensibilidad sobre un rango de variaciones de precios del diesel y del gas natural para comparar dichas opciones con las tecnologías renovables. Se encontró que el sistema híbrido eólico/fotovoltaico/biogás fue ventajoso mientras los costos de producción del biogás no excedieran a 0.1 $\mathrm{USD} / \mathrm{m}^{3}$.

Otros autores investigaron la influencia del cambio climático en el comportamiento técnico y económico de las tecnologías renovables. En particular, $\mathrm{Wu}$ et al. (2016) realizaron un estudio sobre la influencia de dichos cambios en los sistemas fotovoltaicos instalados en techos en Australia. Ellos encontraron que los sistemas fotovoltaicos podrían tener buenos desempeños técnicos, económicos y ambientales hasta el año 2030 para la mayoría de las ciudades australianas; pero podrían disminuir hasta los años 2070 por influencia del cambio climático. Con un enfoque similar, Baek et al. (2016) realizaron un estudio para una de las ciudades más pobladas de Corea del Sur; lo anterior, con el fin de evaluar la viabilidad del uso de sistemas híbridos eólico/fotovoltaicos para la generación de electricidad. El estudio demostró que el uso de tales tecnologías resultaba viable para el suministro eléctrico a grandes distritos y parques industriales de la ciudad, aumentando su independencia energética y mitigando la emisión de gases de efecto invernadero.

Como se puede observar, el estudio de sistemas fotovoltaicos interconectados a red permanece como una preocupación de muchos investigadores en el mundo. Sin embargo, en México no abundan las investigaciones en dicho tema, en particular, no se han estudiado los posibles efectos de la reforma energética en la viabilidad de tales sistemas. El propósito de esta investigación fue realizar la simulación, optimización y el estudio de sensibilidad de diferentes topologías de sistemas fotovoltaicos, enlazados a la red eléctrica mediante un caso de estudio en la Universidad Politécnica del Estado de Guerrero (UPEG), utilizando para ello el modelo de optimización HOMER v2.81. Se realizaron estudios de sensibilidad para determinar la influencia de las siguientes variables en el sistema óptimo: costos capitales y de reemplazamiento de los módulos FV, precio de la electricidad comprada a la compañía eléctrica, tasa de interés anual, carga promedio de consumo y radiación solar promedio diaria. Las tres primeras variables se incluyeron teniendo en cuenta que la Reforma Energética, aprobada por el Gobierno de México podría causar en ellas modificaciones importantes.

\section{Desarrollo}

Para realizar la simulación del sistema fotovoltaico enlazado a la red eléctrica en la Universidad Politécnica del Estado de Guerrero (UPEG), se utilizó el programa HOMER v2.81. Este modelo se empleó en estudios similares, debido a las oportunidades que brinda para realizar simulaciones, optimizaciones y análisis de sensibilidad; que lo hace ventajoso en relación con otros modelos disponibles.

El HOMER es un programa de optimización de sistemas de generación de energía eléctrica, que puede incluir tanto tecnologías renovables como convencionales. Con él es posible realizar tres actividades funda- 
mentales: simulación, optimización y análisis de sensibilidad.

En el proceso de simulación se modela el funcionamiento de una configuración de sistema, en particular, cada hora del año, para determinar su viabilidad técnica y su costo en la vida útil del proyecto. En el de optimización se simulan muchas configuraciones diferentes, buscando aquella que satisfaga las restricciones técnicas al menor costo total presente neto (NPC), que es la función utilizada por el HOMER para ordenar todas las configuraciones posibles. El NPC se determina mediante la ecuación 1 (Lambert et al. 2006).

$N P C=C_{A, T}\left[\frac{(1+i)^{N}-1}{i(1+i)^{N}}\right]$

donde

$C_{A T}=$ costo anualizado total

$i=$ tasa de interés real $\mathrm{y}$

$N=$ tiempo de vida del proyecto

El NPC toma en cuenta todos los costos e ingresos que tienen lugar durante el tiempo de vida del proyecto, expresándolos en valores actuales mediante la tasa de descuento que se fija por el diseñador. Se incluyen los costos de construcción inicial, reemplazamiento de componentes, mantenimiento, combustible, compra de energía a la red y puede incluir costos por penalizaciones a la emisión de gases de efecto invernadero. Los ingresos incluyen los valores residuales de los diferentes componentes del sistema al finalizar su vida útil, así como los ingresos provenientes de la venta de energía a la red.

Entre tanto, el análisis de sensibilidad HOMER lo realiza mediante múltiples optimizaciones bajo un rango de supuestos cambios en variables de entrada (ej. costos de componentes, demanda de energía, radiación solar promedio diaria, velocidad media del viento, tasa de interés, tiempo de vida del proyecto o de componentes, entre otras). Ello permite estimar los efectos que dichos cambios podrían provocar en la configuración del sistema energético óptimo (Lambert et al. 2006).

En este caso de estudio la configuración del sistema FV analizado se presenta en la figura 1.

El sistema incluye un arreglo fotovoltaico, un convertidor, la carga eléctrica y la red de distribución. El costo capital de los módulos FV se consideró igual a $2500 \mathrm{USD} / \mathrm{kW}$, mientras que para el convertidor fue de 800 USD kW; correspondiendo ambos al precio promedio que se comercializa en México. El tiempo de vida del proyecto se tomó igual a 20 años.
El espacio de búsqueda para el arreglo fotovoltaico incluyó los siguientes valores de potencia: $50 \mathrm{~kW}, 60$ $\mathrm{kW}, 70 \mathrm{~kW}, 80 \mathrm{~kW}, 90 \mathrm{~kW}$ y $100 \mathrm{~kW}$. Por otro lado, para el convertidor los valores considerados fueron $70 \mathrm{~kW}$, $80 \mathrm{~kW}, 90 \mathrm{~kW}, 100 \mathrm{~kW}, 110 \mathrm{~kW}, 120 \mathrm{~kW}$ y 130 kW.

Los datos de radiación solar (figura 2), se obtuvieron vía internet a través del HOMER, donde se seleccionó previamente la zona horaria y se introdujeron los datos de latitud y longitud del sitio de estudio. La radiación solar promedio diaria es de $5.82 \mathrm{kWh} / \mathrm{m}^{2}$, mientras que el índice de claridad es de 0.61 .

Los datos de consumo de energía eléctrica se obtuvieron a partir de mediciones realizadas en el actual edificio de la Universidad, utilizando un analizador de calidad de energía (figura 3). La potencia pico es de 71 $\mathrm{kW}$ y el consumo promedio diario es de $494 \mathrm{kWh}$.

Se realizaron estudios de sensibilidad para determinar la influencia que pudieran tener las siguientes variables en el sistema óptimo: radiación solar promedio diaria, carga promedio de consumo, costos capitales y de reemplazamiento de los módulos FV; precio de la electricidad comprada a la red y tasa de interés anual.

Las tres últimas variables se incluyeron teniendo en cuenta que la Reforma Energética, aprobada recientemente por el Gobierno de México, podría causar en ellas modificaciones importantes. Además, con dicho análisis se podrían identificar oportunidades que, en términos de políticas públicas, podrían aumentar la competitividad de los sistemas FV enlazados a la red y con ello potenciar su mayor participación en la matriz energética nacional.

Los valores considerados para la tasa de interés $(12 \%, 9 \%, 6 \%$ y $3 \%)$ tomaron como referencia aquellas donde el Fideicomiso para el Ahorro de Energía Eléctrica (FIDE) otorga créditos para el desarrollo de sistemas fotovoltaicos en el país (entre el 9\% y 12\% aproximadamente, calculados como la Tasa de Interés Interbancaria de Equilibrio más 5 puntos). Los intereses se calculan sobre los saldos insolutos y el reembolso del préstamo se debe realizar en un plazo máximo de 5 años (Fideicomiso para el Ahorro de Energía Eléctrica, 2016).

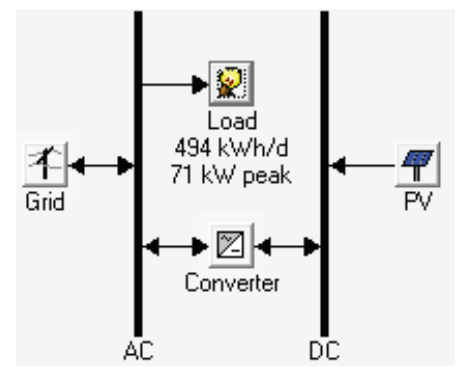

Figura 1. Esquema del sistema FV estudiado 


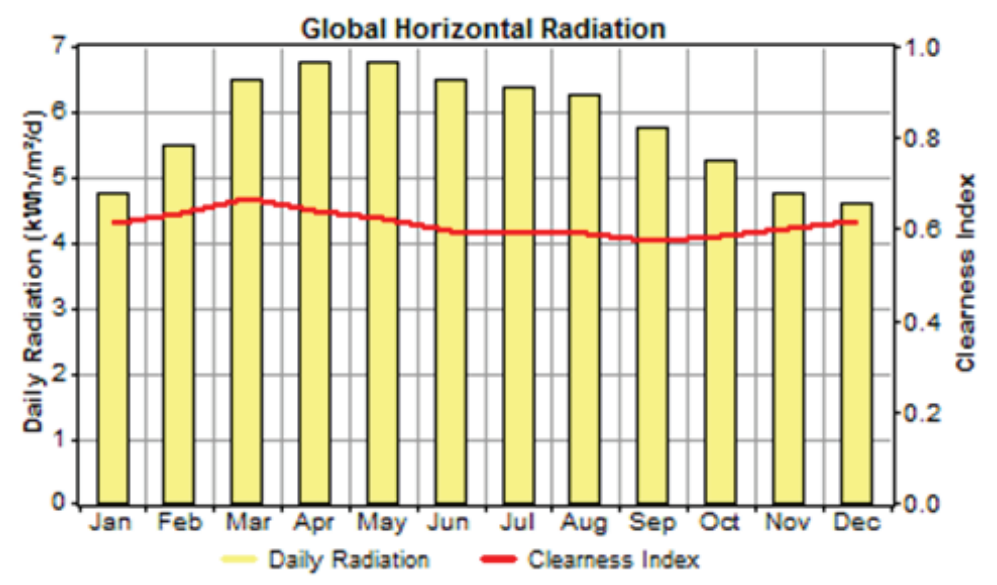

Figura 2. Radiación solar promedio diaria e índice de claridad en el sitio de estudio

Entre tanto, los valores considerados para los precios de la electricidad comprada con la red, variaron entre 0.1USD/kWh y $0.5 \mathrm{USD} / \mathrm{kWh}$. Esto se realizó con la doble intención de: a) que los resultados se compararan con los obtenidos por otros autores en distintos estudios, donde utilizan valores parecidos; b) teniendo conocimiento que en México el precio de la electricidad para la tarifa HM (a la cual se le factura el servicio eléctrico a la UPEG) es inferior a $0.3 \mathrm{USD} / \mathrm{kWh}$, determinar qué variaciones tendrían que sufrir las tasas de interés y los precios de los sistemas fotovoltaicos para que estos resulten competitivos a bajos precios de la electricidad comprada a CFE.

\section{Resultados y Discusión}

En la figura 4 se muestran los resultados del proceso de simulación y optimización económica (basada en el costo presente neto), para los valores actuales tanto del consumo de electricidad en la UPEG, como de los costos de los módulos FV, considerando una tasa de interés de $6 \%$ y un precio de la energía comprada a la compañía de electricidad igual a $0.3 \$ / \mathrm{kWh}$.

Como se puede observar, el sistema óptimo atendiendo al valor de su Costo Neto Presente (CNP), es aquel constituido por $100 \mathrm{~kW}$ de un arreglo FV y un convertidor de $80 \mathrm{~kW}$, enlazado a la red eléctrica. Dicho sistema

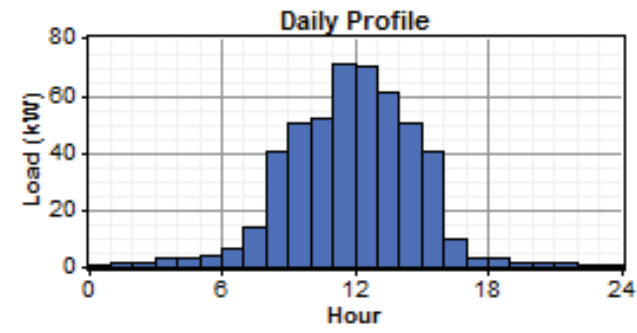

Figura 3. Perfil promedio diario de carga podría suministrar $176497 \mathrm{kWh} / \mathrm{año}$, de los cuales 28642 $\mathrm{kWh} / \mathrm{año}$ se venden a la red eléctrica y el resto se suministran al consumo (que es de 180310 kWh/año), lo cual representa $82 \%$ de la demanda de electricidad de la UPEG; a un costo nivelado de $0.20 \$ / \mathrm{kWh}$. Por otro lado, un sistema FV con una potencia instalada igual a la mitad $(50 \mathrm{~kW})$ de la del sistema óptimo, aportaría $47 \%$ del consumo a un costo nivelado ligeramente superior $(0.27 \$ / \mathrm{kWh})$.

Sin embargo, si como consecuencia de la Reforma Energética, el precio de la electricidad comprada a la red convencional disminuye por debajo de los 0.25 \$/ $\mathrm{kWh}$ (para una tasa de interés de $6 \%$ y un costo del kW FV de $2500 \$ / \mathrm{kW}$ ) sería preferible consumir electricidad de la red en lugar de instalar un sistema FV interconectado a ella (figura 5).

Lo anterior se puede observar con mayor claridad en la figura 6, donde se muestra el espacio de sensibilidad para diferentes valores del precio de la electricidad comprada desde la red y del consumo promedio diario de electricidad en la UPEG.

El consumo actual promedio diario de electricidad en la UPEG es de $494 \mathrm{kWh}$, pero se espera que aumente, ya que es una Universidad donde se construyen nuevos edificios, los cuales recibirán una mayor cantidad de estudiantes. Debido a esto, se realizaron los cálculos para consumos superiores al actual. Los resultados indican (figura 6) que si el precio de la electricidad comprada a la compañía de electricidad disminuyera por debajo de los $0.25 \mathrm{USD} / \mathrm{kWh}$, el sistema óptimo sería la red convencional de electricidad, independientemente del aumento en el consumo promedio diario de electricidad en la UPEG. Es decir, el sistema fotovoltaico dejaría de ser competitivo frente a la red eléctrica convencional.

Ante dicho escenario podrían existir otras opciones para hacer competitivos los sistemas FV, en este caso: 
a) Disminución de las tasas de interés

b) Disminución de los costos FV

Estos dos factores podrían tener lugar con la implementación de la Reforma Energética en México. Se analizan los resultados (figuras 7 y 8).

En la figura 7 se observa, que si el precio de la energía disminuye por debajo de $0.25 \mathrm{USD} / \mathrm{kWh}$ y la tasa de interés es mayor a $3 \%$, el sistema óptimo es la red eléctrica convencional; el sistema FV solo competiría si la tasa de interés disminuyera por debajo de $3 \%$.

Por otro lado, si vemos el espacio de sensibilidad para diferentes valores del precio de la energía y del costo de los módulos FV (figura 8), se puede notar que por debajo de 0.2 USD/kWh y una disminución del precio de los módulos no mayor a 50\% el sistema óptimo será la red convencional, si la tasa de interés es de $12 \%$. Sin embargo, si la tasa de interés disminuye a $6 \%$ el sistema FV enlazado a la red gana espacio, dejando de ser óptimo solo por debajo de $0.15 \mathrm{USD} / \mathrm{kWh}$.

En cuanto al período de recuperación de la inversión, este es uno de los indicadores económicos que se vería modificado, entre otros factores, por los cambios en la tasa de interés, en el precio de la energía comprada con la compañía de electricidad y en los costos de la tecnología fotovoltaica.

Por ejemplo, para el caso del sistema FV de $100 \mathrm{~kW}$ que es el sistema óptimo en la figura 4 (0.3 USD/kWh,

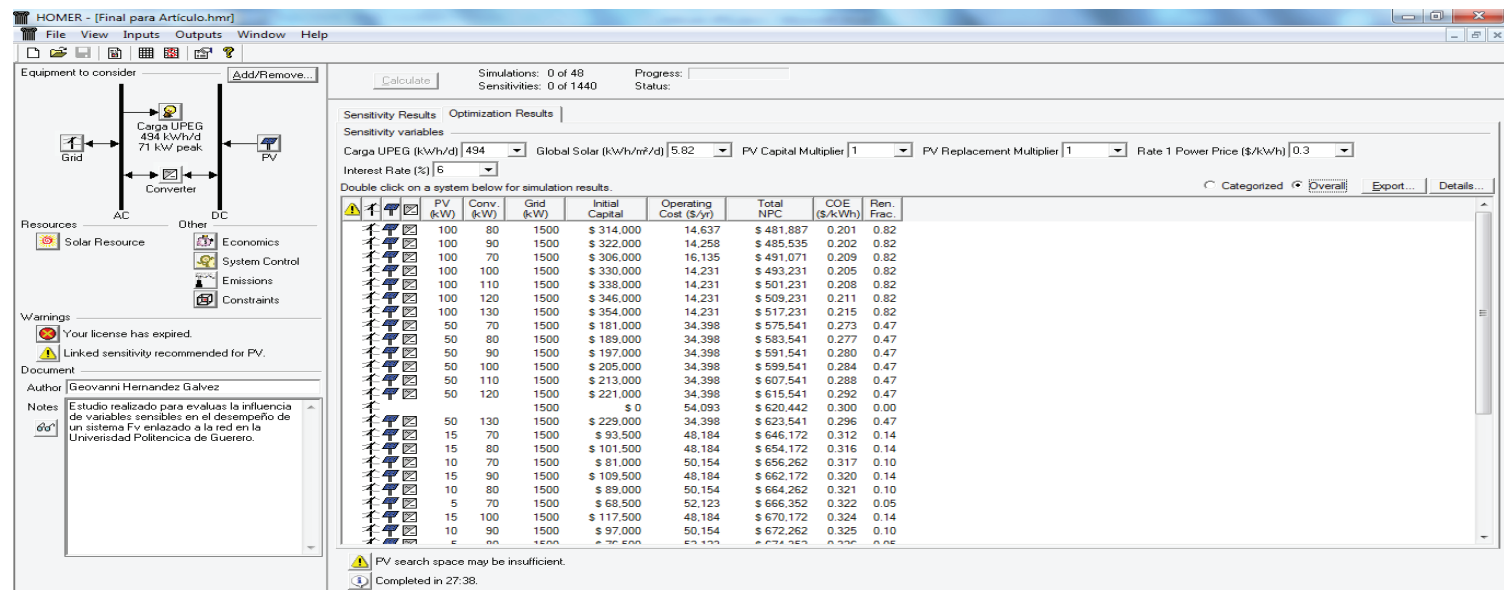

Figura 4. Resultados de la simulación y optimización del sistema estudiado para 0.3 \$/kWh (PV es la capacidad del sistema fotovoltaico; Conv. es la capacidad del convertidor; Grid es la capacidad de la red eléctrica; Initial Capital es la inversión inicial; Operating Cost es el costo de operación anual; Total NPC es el costo presente neto total; COE es el costo de la energía y Ren. Frac. es la fracción de energía que es suministrada por las tecnologías renovables)

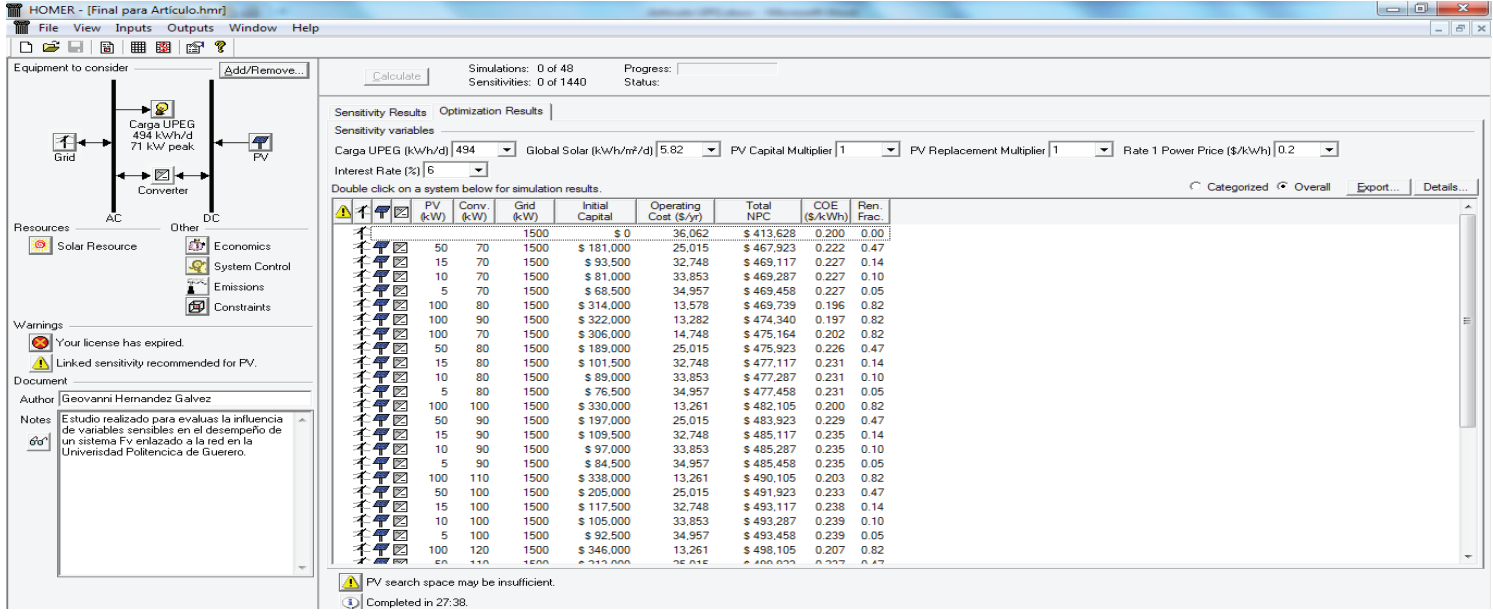

Figura 5. Resultados de la simulación y optimización del sistema estudiado para 0.2 \$/kWh 
$6 \%$ de tasa de interés), el período de recuperación de la inversión es de 11 años (figura 9), por lo que se contaría con 9 años o más de beneficios netos (considerando que el sistema FV podría tener una vida útil de 20 años e incluso un poco más). Sin embargo, para una tasa de interés de $9 \%$ la inversión se recuperaría en casi 15 años, por otro lado, si es de 3\% disminuiría a 9 años.

Es de resaltar que los períodos de recuperación, antes mencionados son relativamente elevados, sobre todo si se compara con el período de 5 años que establece el FIDE para la devolución de los préstamos. Debido a ello se deben implementar medidas que permitan que el mercado energético evolucione hacia una disminución en los períodos de recuperación de la inversión de los sistemas FV.
Una de dichas medidas podría dirigirse a lograr una disminución en los precios de venta de los componentes fotovoltaicos, fundamentalmente de los módulos. Como se puede observar en la figura 11, si el costo de los módulos disminuye 50\%, el sistema FV óptimo (figura 10) tendría un período de recuperación de la inversión de 6.5 años.

Obviamente el efecto combinado de la disminución en el costo FV y en las tasas de interés, sería una disminución no significativa en el período de recuperación (5.8 años para $6 \%$ de tasa de interés y 5 años para $3 \%$ ).

Finalmente, analizamos cómo afectarían al espacio de sensibilidad, las variaciones en la radiación solar promedio diaria. Como se puede observar en la figura 12, para los costos actuales de los módulos fotovoltai-

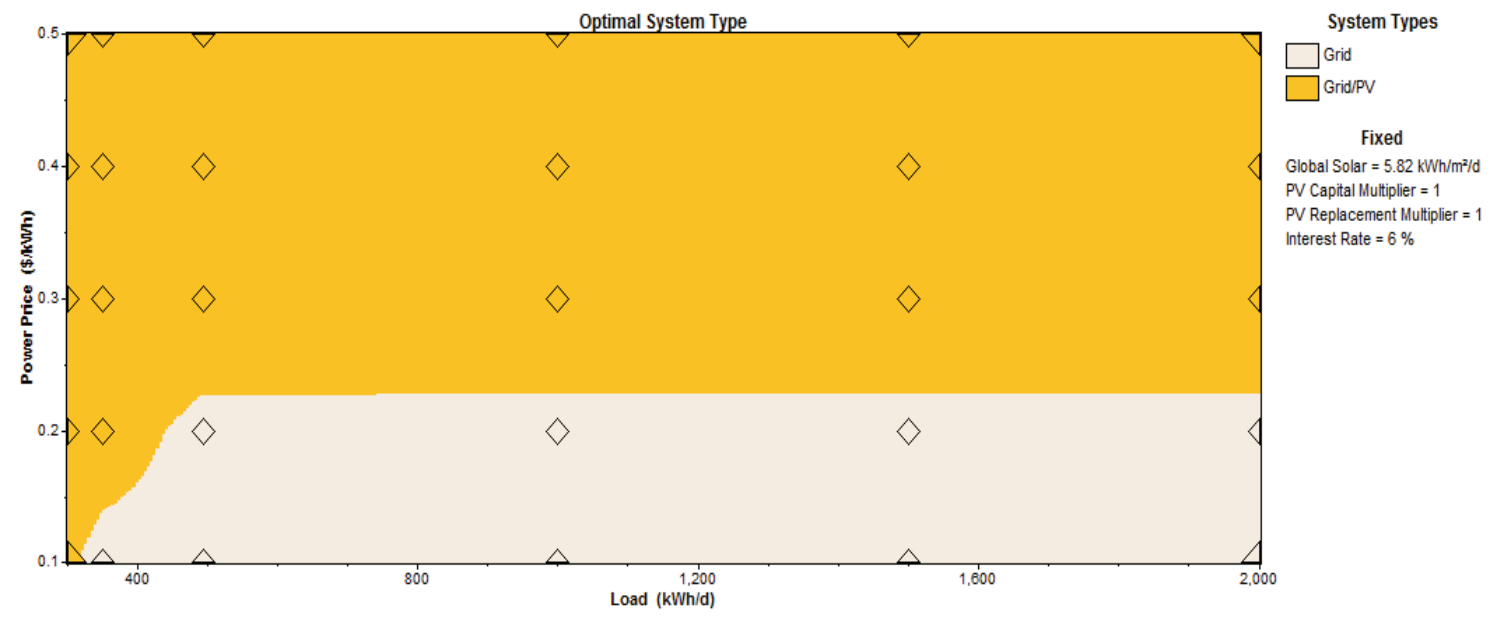

Figura 6. Espacio de sensibilidad para diferentes valores del precio de la electricidad comprada desde la red y del consumo promedio diario de electricidad en la UPEG

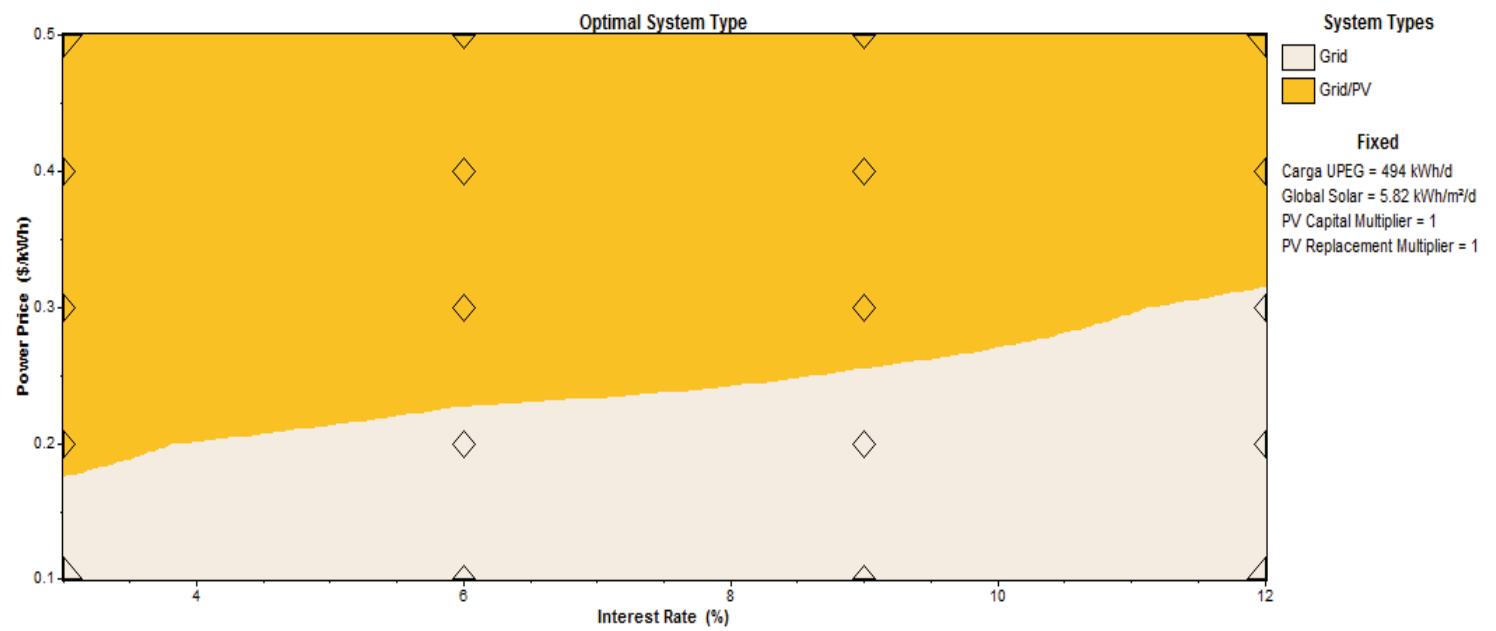

Figura 7. Espacio de sensibilidad para diferentes valores de la tasa de interés y del precio de la electricidad comprada desde la red 

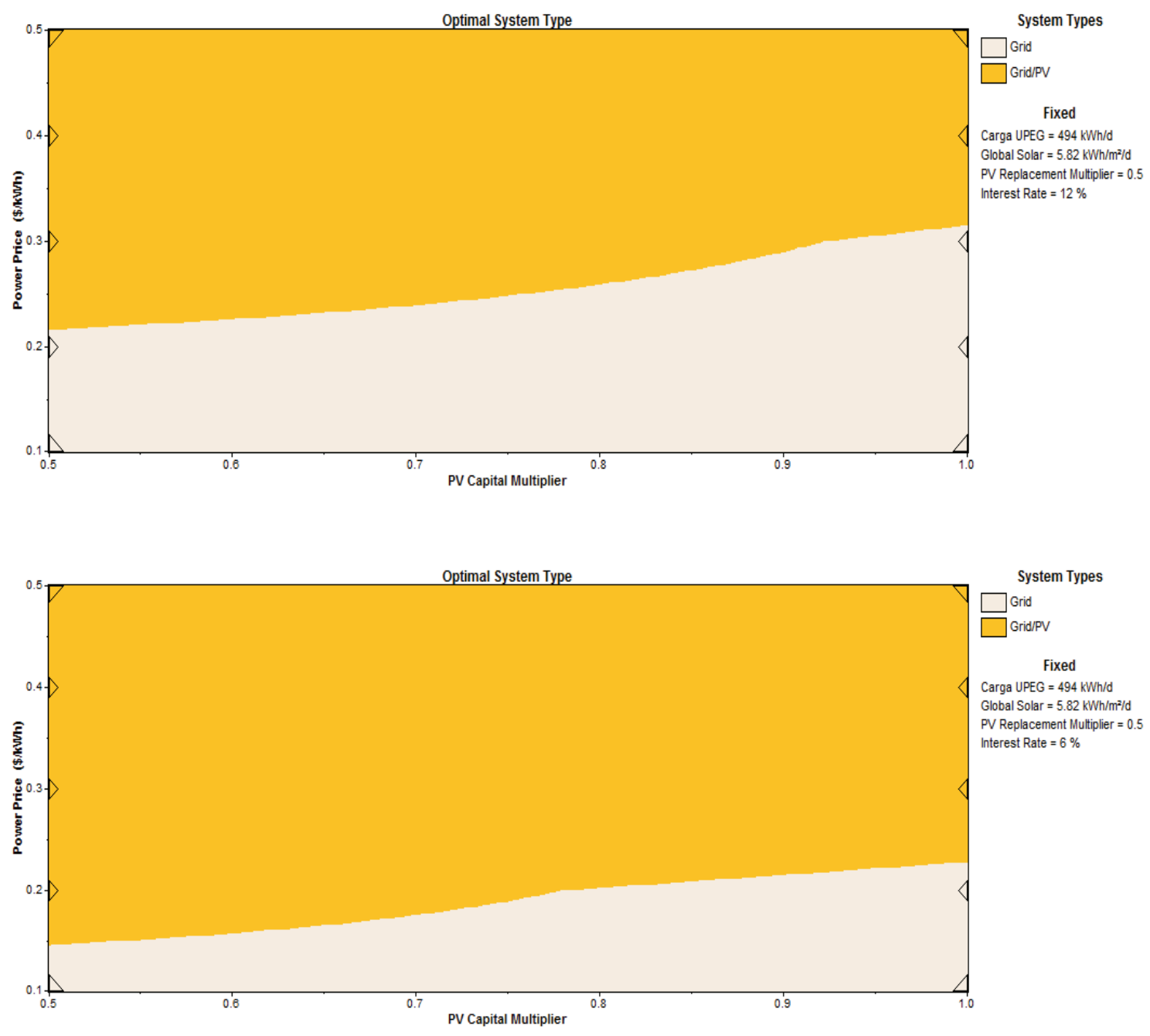

Figura 8. Espacios de sensibilidad para diferentes valores del costo de los módulos fotovoltaicos y del precio de la electricidad comprada desde la red para tasas de interés de $12 \%$ y $6 \%$

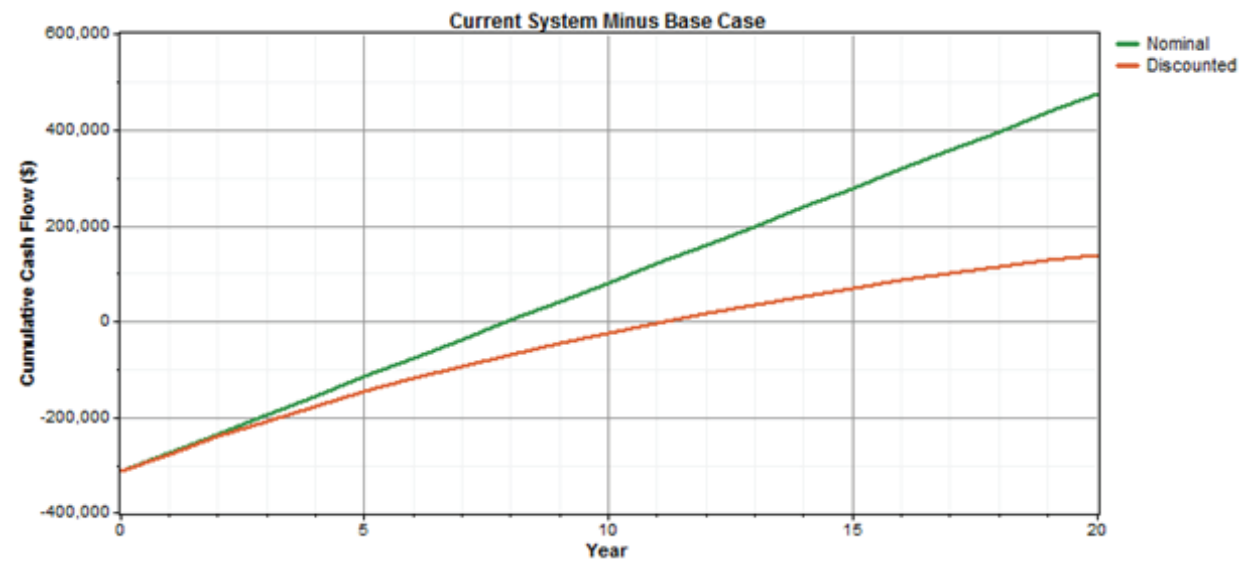

Figura 9. Período de recuperación de la inversión $(0.3$ USD/kWh, 6\% de tasa de interés) 


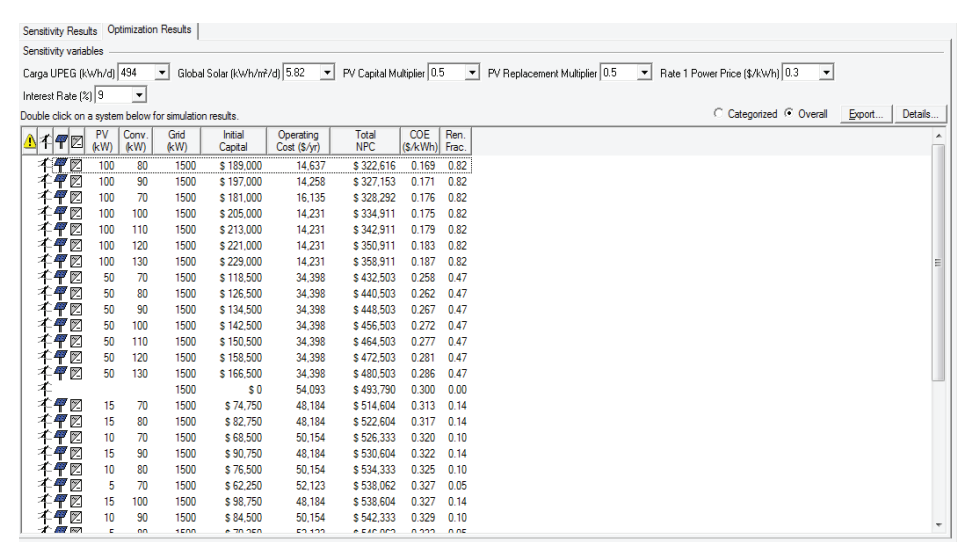

Figura 10. Resultados de la simulación y optimización del sistema estudiado $(0.3$ USD/kWh, 9\% de tasa de interés y 50\% de disminución del costo de los módulos FV)

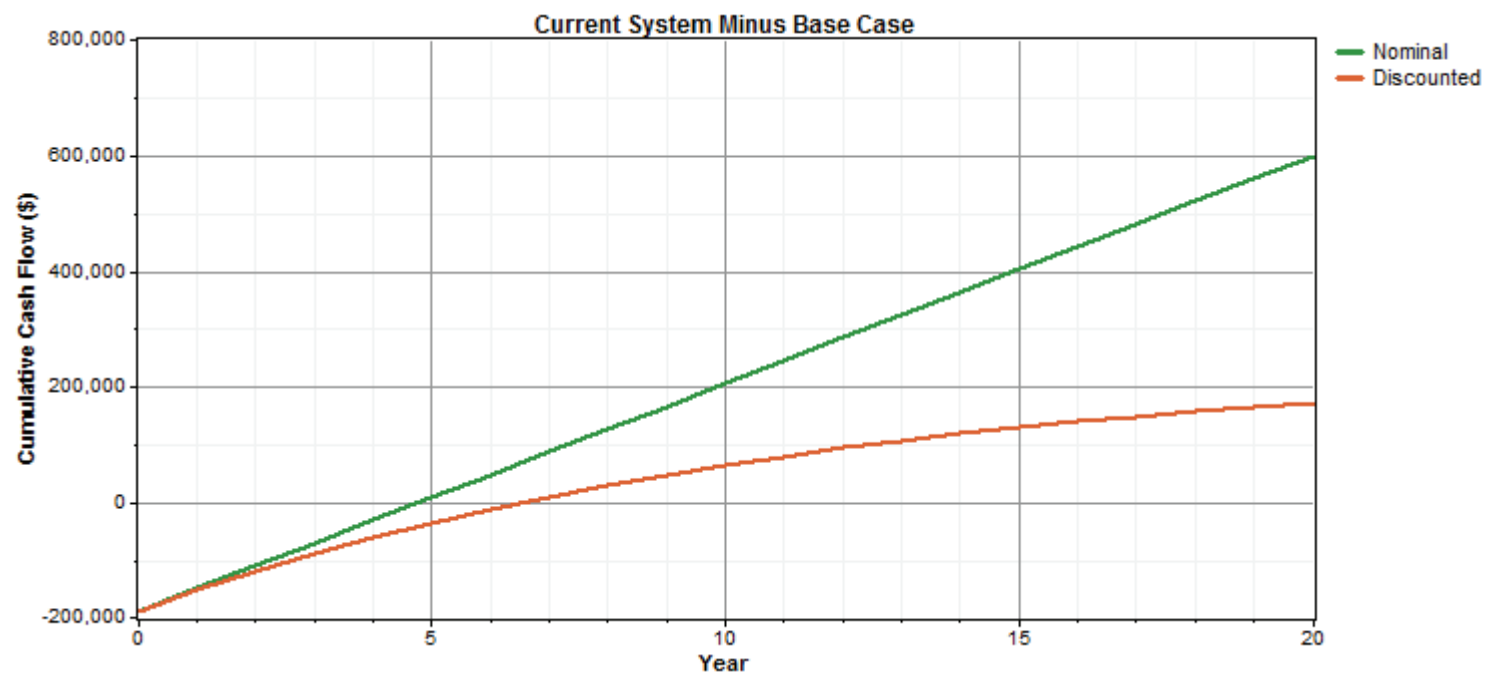

Figura 11. Período de recuperación de la inversión (0.3 USD/kWh, 9\% de tasa de interés y 50\% de disminución del costo de los módulos FV)

cos (PV capital multiplier $=1), 0.3 \mathrm{USD} / \mathrm{kWh}$ de costo de la electricidad comprada a la red y $9 \%$ de tasa de interés, el sistema FV enlazado a la red deja de ser el óptimo para valores de radiación solar inferiores a 5 $\mathrm{kWh} / \mathrm{m}^{2} / \mathrm{d}$ en casi todo el espacio de búsqueda del consumo promedio diario de electricidad.

En cambio, para las mismas condiciones, pero con una disminución de $50 \%$ en el costo de los módulos FV (PV capital múltiple igual a 0.5), el sistema FV gana espacio frente a la red convencional, donde el sistema óptimo aún para valores de la radiación solar promedio diaria son arriba de los $3.7 \mathrm{kWh} / \mathrm{m}^{2}$. Esto haría que dichos sistemas pudieran ser idóneos para una mayor cantidad de sitios, aunque no cuenten con los niveles de radiación solar que existen en la UPEG. Lo anterior también aplica si el costo de la electricidad comprada a la compañía se incrementara (ej. $0.4 \mathrm{USD} / \mathrm{kWh}$, figura
13, como sucede en México), en vez de disminuir una vez implementada la reforma energética.

Los resultados descritos anteriormente demuestran la influencia de algunas variables en el espacio de sensibilidad, en ciertos indicadores económicos como el período de recuperación de la inversión y en el tipo de sistema energético óptimo. Esto ayuda a identificar acciones en materia de políticas públicas para potenciar el uso de sistemas FV enlazados a la red en México.

\section{Conclusiones}

Los resultados del estudio demuestran que un sistema fotovoltaico de $100 \mathrm{~kW}$ enlazado a la red eléctrica en la UPEG, podría suministrar $82 \%$ de su consumo de electricidad a un costo competitivo (0.2 \$USD $/ \mathrm{kWh}$ ) en relación con la proveniente de la red convencional $(0.3$ 

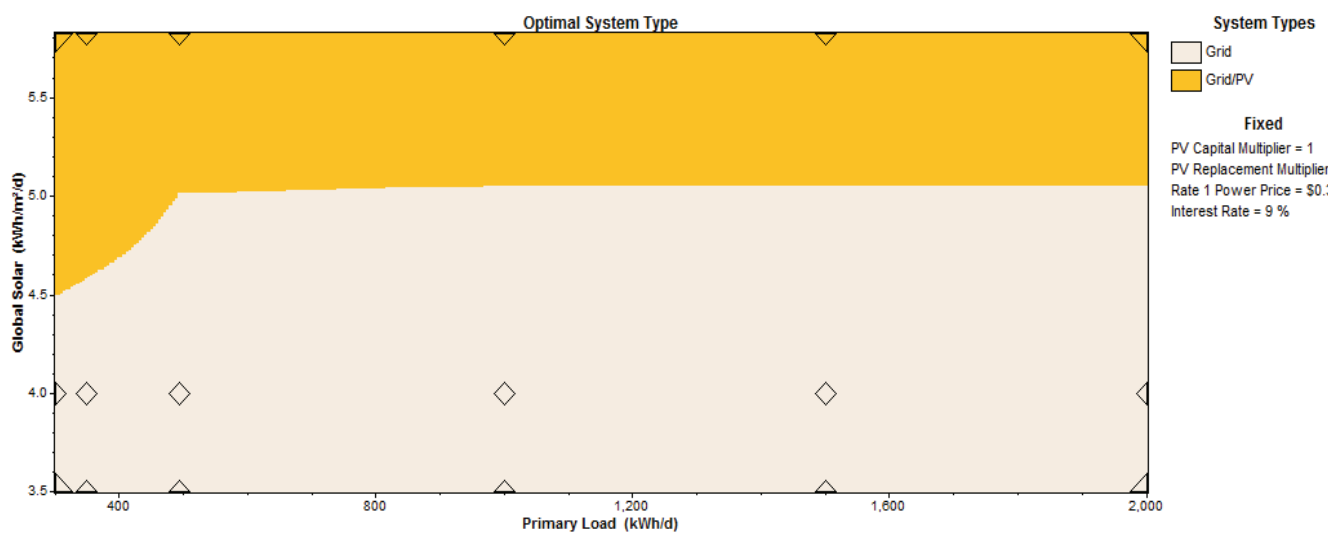

PV Capital Muttiplier $=1$

PV Replacement Muttiplier $=1$

Rate 1 Power Price $=\$ 0.3 / \mathrm{kWh}$

Interest Rate $=9 \%$

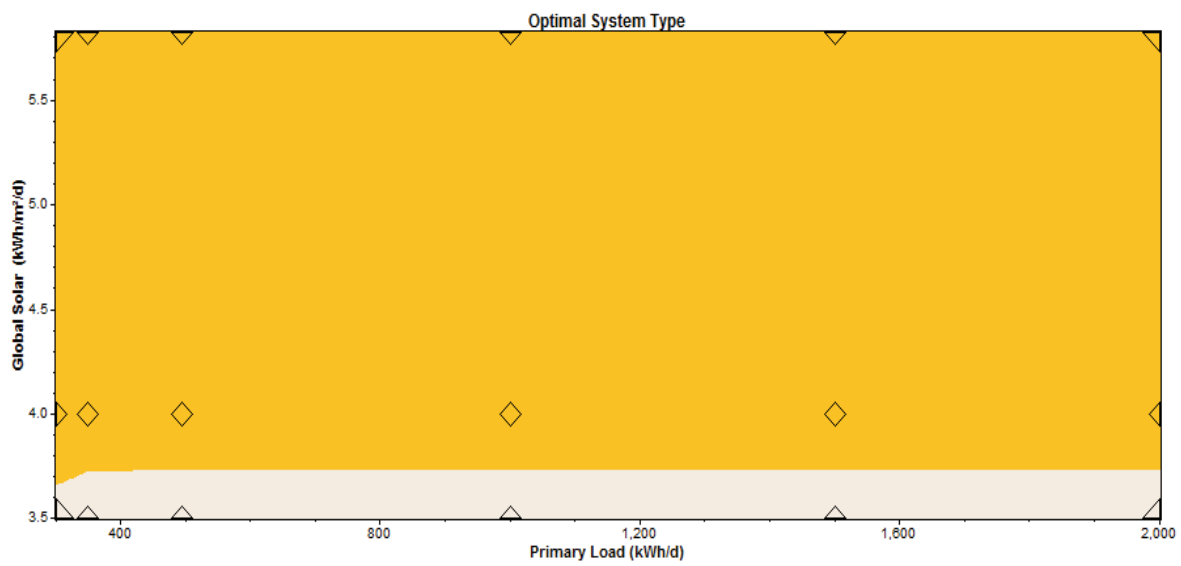

System Types

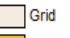

$\square$ Grid/PV

Fixed
PV Capital Muttiplier $=0.5$

PV Replacement Muttiplier $=0.5$

Rate 1 Power Price $=\$ 0.3 / \mathrm{kWh}$

Interest Rate $=9 \%$

Figura 12. Espacios de sensibilidad para diferentes valores de la radiación solar promedio diaria y del consumo promedio diario (multiplicadores de capital de los módulos FV iguales a 1 y a 0.5, respectivamente)

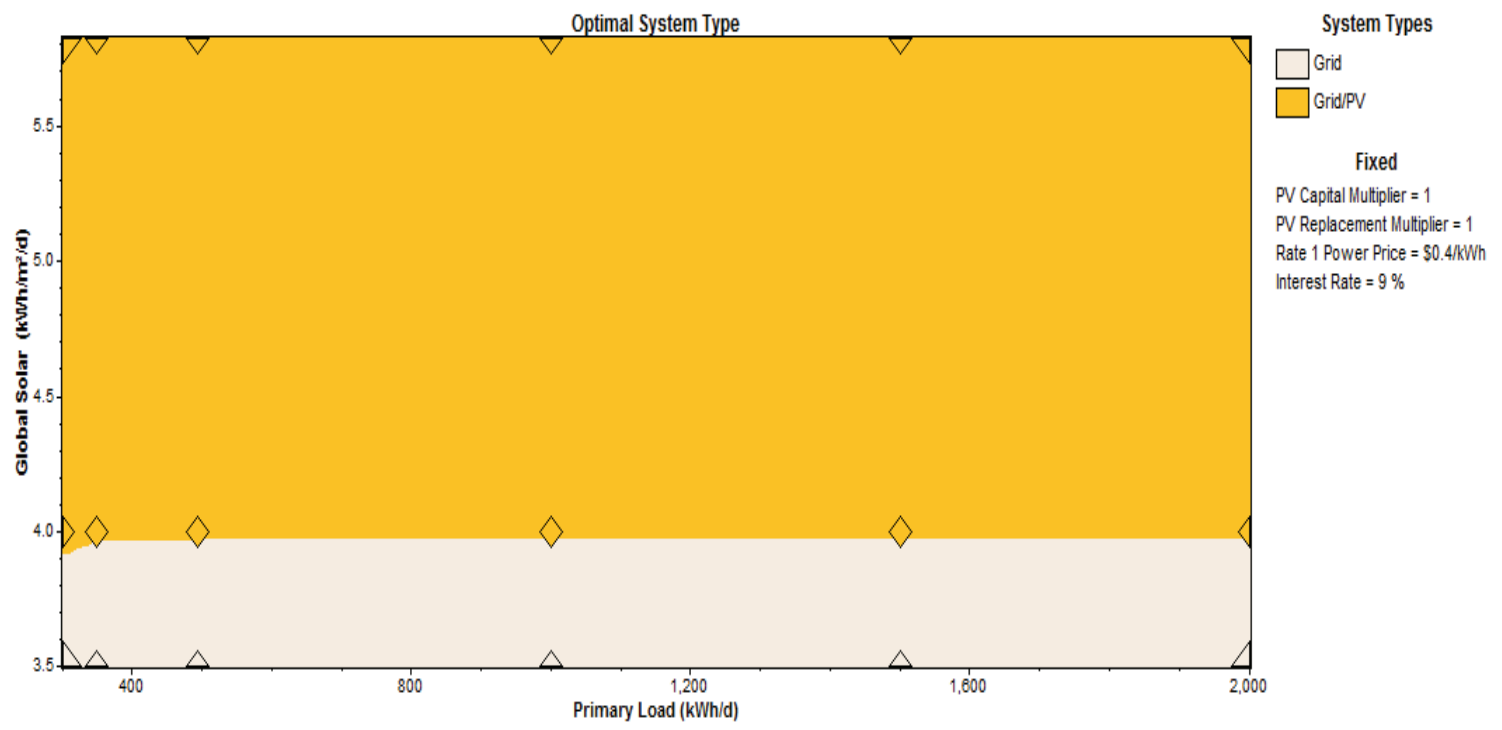

Figura 13. Espacio de sensibilidad para diferentes valores de la radiación solar promedio diaria y del consumo promedio diario (multiplicadores de capital de los módulos FV iguales a 1, tasa de interés $9 \%$ y precio de la electricidad 0.4 USD/kWh) 
$\$ \mathrm{USD} / \mathrm{kWh}$ ). En este caso, para $6 \%$ de tasa de interés el PR de la inversión sería de 11 años, mientras que si el costo de los módulos FV disminuye un 50\% se alcanzarían PR de 5 a 6.5 años. Esta disminución en costos haría a los sistemas $\mathrm{FV}$ competitivos aún para valores de la radiación solar promedio diaria igual a $3.7 \mathrm{kWh} / \mathrm{m}^{2}$, que es menor a la disponible en la UPEG; lo que facilitaría la diseminación de tales sistemas en México y en particular, en el estado de Guerrero. Por otro lado, si como resultado de la Reforma Energética Constitucional en México, los precios de la electricidad vendida por la compañía de electricidad disminuyeran por debajo de $0.25 \$ \mathrm{USD} / \mathrm{kWh}$, la alternativa fotovoltaica dejaría de ser competitiva. Ante tal escenario, el mercado fotovoltaico en el país deberá evolucionar hacia una disminución en los costos de los componentes fotovoltaicos, principalmente de los módulos, y en las tasas de interés. La falta actual de una cantidad suficiente de proveedores de tales componentes en el estado de Guerrero, es una de las debilidades que atentan contra la diversificación de dicha tecnología. A esto se suman otras barreras determinadas por la escasa actividad de investigación básica y aplicada en el área de las energías renovables en el estado, escasez de programas educativos relacionados con las fuentes renovables de energía, falta de vinculación entre las instituciones educativas y las empresas y la escasez de recursos humanos calificados, en el estado en el área de las fuentes renovables de energía. Por ello, las políticas energéticas deberán atender de manera prioritaria estas necesidades.

\section{Agradecimientos}

Se agradece al programa PROMED por su apoyo con el financiamiento del proyecto: “Optimización multicriterio de sistemas energéticos renovables: aplicación a un sistema fotovoltaico de $4 \mathrm{~kW}$ enlazado a la red en la Universidad Politécnica del Estado de Guerrero" (Número de oficio de la carta de liberación: PROMED/ 103-5/12/8160); gracias al cual fue posible realizar esta investigación.

\section{Referencias}

Baek S., Park E., Min-Gil K., Jib-Kwon S., Ki-Joon K., Ohm J.Y., Del Pobil A.P. Optimal renewable power generation systems for Busan metropolitan city in South Korea. Renewable Energy [en línea], volumen 88, 2016: 517-525. Disponible en: http://dx. doi.org/10.1016/j.renene.2015.11.058.

Bronski P., Creyts J., Guccione L., Madrazo M., Mandel J., Rader B., Seif D., Lilienthal P., Glassmire J., Abromowitz J., Crowdis M., Richardson J., Schmitt E., Tocco H. The economics of grid de- fection: when and where distributed solar generation plus storage competes with traditional utility service. Rocky Mountain Institute, Boulder, Colorado, 2014 [en línea]. Disponible en: http://www.rmi.org/electricity_grid_defection.

Bronski P., Creyts J., Crowdis M., Doig S., Glassmire J., Guccione L., Lilienthal P., Mandel J., Rader B., Seif D., Tocco H., Touati $\mathrm{H}$. The economics of load defection: how grid-connected solar-plusbattery systems will compete with traditional electric service, why it matters, and possible paths forward. Rocky Mountain Institute, Boulder, Colorado, 2015 [en línea]. Disponible en: http://www.rmi.org/electricity_load_defection.

Fideicomiso para el Ahorro de Energía Eléctrica. Programas Sustantivos. Eficiencia Energética. Disponible en: http://www. fide.org.mx/index.php?option=com_content\&view=article\&i $\mathrm{d}=121 \&$ Itemid $=219$.

Getachew B.y Björn P. Feasibility study for a standalone solarwind-based hybrid energy system for application in Ethiopia. Applied Energy [en línea], volumen 87, (número 2), 2010: 487-495. doi:10.1016/j.apenergy.2009.06.006. Disponible en: Journal homepage: www.elsevier.com/locate/apenergy.

International Energy Agency. Key World Energy Statistics, 2014 [en línea]. Disponible en: http://www.oecd-ilibrary.org/energy/key-world-energy-statistics_22202811.

International Energy Agency. Trends 2015 in photovoltaic applications. Survey report of selected IEA countries between 1992 and 2014 [en línea]. Disponible en: http://www.iea-pvps.org/ fileadmin/dam/public/report/national/IEA-PVPS_-_ Trends_2015_-_MedRes.pdf.

Kalinci Y. Alternative energy scenarios for Bozcaada island, Turkey. Renewable and Sustainable Energy Reviews [en línea], volumen 45, 2015: 468-480. Disponible en: http://dx.doi. org/10.1016/j.rser.2015.02.001.

Khalilpour R. y Vassallo A. Leaving the grid: An ambition or a real choice? Energy Policy [en línea], volumen 82, 2015: 207-221. Disponible en: http://dx.doi.org/10.1016/j.enpol.2015.03.005.

Lambert T., Gilman P., Lilienthal P. Micropower system modeling with HOMER [en línea], Disponible en: http://www.homerenergy.com/documents/MicropowerSystemModelingWithHOMER.pdf

Mudasser M., Yiridoe E.K., Corscadden K. Cost-benefit analysis of grid-connected wind-biogas hybrid energy production, by turbine capacity and site. Renewable Energy [en línea], volumen 80, 2015: 573-582. Disponible en: http://dx.doi.org/10.1016/j.renene.2015.02.055.

Ramli-Makbul A.M., Hiendro A., Sedraoui K., Twaha S. Optimal sizing of grid-connected photovoltaic energy system in Saudi Arabia. Renewable Energy [en línea], volumen 75, 2015: 489-495. Disponible en: http://dx.doi.org/10.1016/j.renene.2014.10.028.

Saheb-Koussa D., Koussa M., Belhamel M., Haddadi M. Economic and environmental analysis for grid-connected hybrid photovoltaic-wind power system in the arid region. Energy Procedia 
[en línea], volumen 6, abril 2011: 361-370. doi:10.1016/j.egypro.2011.05.042. Disponible en: www.sciencedirect.com.

Sarker S. Feasibility analysis of a renewable hybrid energy system with producer gas generator fulfilling remote household electricity demand in Southern Norway. Renewable Energy [en línea], volumen 87, 2016: 772-781. Disponible en: http://dx.doi. org/10.1016/j.renene.2015.11.013.

Velo R., Osorio L., Fernández M.D., Rodríguez M.R. An economic analysis of a stand-alone and grid-connected cattle farm. Renewable and Sustainable Energy Reviews [en línea], volumen 39, 2014: 883-890. doi: http://dx.doi.org/10.1016/j. rser.2014.07.156. Disponible en: Journal homepage: www. elsevier.com/locate/rser.

Wu-Ma W., Rasul M.G., Liu G., Li M., Hui-Tan X. Climate change impacts on techno-economic performance of roof PV solar system in Australia. Renewable Energy [en línea], volumen 88, 2016: 430-438. Disponible en: http://dx.doi.org/10.1016/j.renene.2015.11.048. 


\section{Citación sugerida:}

\section{Citación estilo Chicago}

Rivera-Martínez, Mario Arturo, Geovanni Hernández-Galvez, Omar Sarracino-Martínez, Luis Ixtlilco-Cortés, Alina Juantorena-Ugás, Ulises Ivan Batalla-Nolasco, David Becerra-García. Análisis de sensibilidad de sistemas fotovoltaicos conectados a la red eléctrica: Caso de estudio en Guerrero, México. Ingeniería Investigación y Tecnología, XVIII, 03 (2017): 293-305.

\section{Citación estilo ISO 690}

Rivera-Martínez M.A, Hernández-Galvez G., Sarracino-Martínez O., Ixtlilco-Cortés L., Juantorena-Ugas A., Batalla-Nolasco U.I, BecerraGarcia D. Análisis de sensibilidad de sistemas fotovoltaicos conectados a la red eléctrica: Caso de estudio en Guerrero, México. Ingeniería Investigación y Tecnología, volumen XVIII (número 3), julio-septiembre 2017: 293-305.

\section{Semblanzas de los autores}

Mario Arturo Rivera-Martínez. Ingeniero químico, maestro en energía solar y doctor en ingeniería y ciencias aplicadas. Es profesor investigador de tiempo completo en la Universidad Politécnica del Estado de Guerrero. Ha dirigido tesis de pregrado y posgrado, es miembro del Registro de Evaluadores del CONACYT. Ha publicado artículos en revistas indizadas y en memorias de congresos. Miembro del Sistema Nacional de Investigadores y del Sistema Estatal de Investigadores del Estado de Guerrero.

Geovanni Hernández-Galvez. Licenciado en física, maestro en energías y doctor en ingeniería. Es miembro del Sistema Nacional de Investigadores, Nivel I. Es profesor titular A en la Universidad Popular de la Chontalpa. Ha participado en varios proyectos de investigación, cuenta con 12 artículos publicados en revistas indexadas y ha participado en alrededor de 16 congresos nacionales e Internacionales. Realiza investigaciones en las áreas de energía eólica y de optimización multicriterio de sistemas híbridos.

Omar Sarracino-Martínez. Doctor en ciencia de los materiales CIMAv-Chihuahua. Profesor investigador de la Universidad Popular de la Chontalpa. Miembro del SNI nivel I. Realiza investigaciones dirigidas al estudio de materiales para el desarrollo de celdas solares en película delgada, así como aplicaciones de tecnologías fotovoltaicas.

Luis Ixtlilco-Cortés. Ingeniero químico, maestro en energía y doctor en ingeniería. Adscrito actualmente a la Universidad Politécnica del Estado de Guerrero. Ha dirigido varias tesis de licenciatura y maestría. Es miembro del registro de evaluadores del CONACYT. Es miembro del Sistema Nacional de Investigadores (SNI-1) desde 2013 y del Sistema Estatal de Investigadores del Estado de Guerrero. Miembro de la Comisión Técnica del área de Ciencias Exactas e Ingeniería del Consejo Académico del COCYTIEG.

Alina Juantorena-Ugás. Licenciada en ingeniería química, maestra en ingeniería de procesos y doctora en ingeniería. Es docente e investigadora en la Universidad Autónoma del Estado de Morelos. Ha dirigido 14 tesis de licenciatura y 3 de maestría. Ha publicado varios artículos en revistas de impacto. Es miembro nivel 1 del SNI y del Sistema Estatal de Investigadores (SEI) del Estado de Morelos.

Ulises Ivan Batalla-Nolasco. Es ingeniero en energías por la Universidad Politécnica del Estado de Guerrero. Actualmente realiza estudios de maestría en la Universidad Autónoma del Estado de Guerrero.

David Becerra-García. Ingeniero mecánico. Maestro en ingeniería y en ciencias aplicadas en opción terminal de tecnología de materiales. Doctor en ingeniería en energía con la especialidad de celdas solares. Es profesor-investigador en la Universidad Politécnica del Estado de Guerrero. Realiza investigaciones con celdas solares, estructuras fotónicas y sensores a base de silicio poroso. Ha publicado varios artículos ligados a sus líneas de investigación. 
DOI: http://dx.doi.org/10.22201/fi.25940732e.2017.18n3.026 